\title{
Numerical Simulation of the Flow of Crowds at the Jamarat Bridge during the Annual Hajj Event
}

\author{
Chokri Mnasri, Abdulsalam Farhat \\ Department of Mathematics, College of Science, King Faisal University, Al-Ahsa, Saudi Arabia \\ Email:cmnasri@kfu.edu.sa, almotatwer@hotmail.com
}

How to cite this paper: Mnasri, C. and Farhat, A. (2016) Numerical Simulation of the Flow of Crowds at the Jamarat Bridge during the Annual Hajj Event. Open Journai of Fluid Dynamics, 6, 321-331. http://dx.doi.org/10.4236/ojfd.2016.64024

Received: September 9, 2016 Accepted: December 4, 2016

Published: December 7, 2016

Copyright $\odot 2016$ by authors and Scientific Research Publishing Inc. This work is licensed under the Creative Commons Attribution International License (CC BY 4.0).

http://creativecommons.org/licenses/by/4.0/

\begin{abstract}
The huge number of pilgrims to the holy Mecca in the Hajj needs high awareness of crowd safety management. The stoning of the Jamarat, which is one of the rituals of the Hajj, undergoes the most dangerous crowd movements where fatal accidents occurred. This work investigates some problems related with the crowd dynamics when stoning the Jamarat pillars and gives some solutions. The main idea of this research is to suppose that the crowd dynamics is assimilated to fluid movement under certain conditions. Numerical simulation using a computational fluid dynamics program is used to solve Navier-Stokes equations governing the mechanics of homogeneous and incompressible fluid in a domain similar to the Jamarat Bridge from the entrance to the middle Jamarah. Some solutions are proposed inspired by the flow solutions to better manage crowd movements in the Jamarat Bridge and eventually in other similar dynamics events like sporting events.
\end{abstract}

\section{Keywords}

Computational Fluid Dynamics, Crowd Dynamics, Crowd Management, Jamarat Bridge

\section{Introduction}

The control and management of large crowds flow is a field of great interest. Interactions between mathematics and applied science in such fields are very strong. A typical example of these crowds is the annual pilgrimage in Makkah city (Hajj).

Pedestrian's movement from the dangerous areas, under several physical constraints, is a challenging problem. If people fail to escape from a building in time, people may be injured and killed. Failure of obstacles dysfunction or wrong exit gate can be the causes of such accidents (Xiaoping et al. [1]).

Many studies and reviews ([2] [3]) of crowd dynamic have been investigated over the 
past years. In particular, Fluid dynamics models ([3]-[8]) are based on the idea that collective movement that is the crowd movement can be likened to the flow of a fluid.

There are two main physical approaches for modeling crowd motion. The first approach is supposing crowd motion similar to discrete individual's motion like granular material behavior. The second approach, applicable only in large crowds, involves treating the crowd as a continuous medium as in fluid mechanics [7].

Henderson has presented a theory of the flow of people along a channel. This theory stipulated that a crowd fluid may exhibit anomalous gas dynamic behavior caused by a change in the sign of a well-known derivative. This indicated that expansion shocks are possible in crowd fluids like real fluids [9].

Helbing et al. have analyzed video recordings of the crowd disaster in Mina/Makkah during the Hajj in 1426H on January 12, 2006 and have revealed two subsequent, sudden transitions from laminar to stop-and-go and "turbulent" flows there to "turbulent" crowd motion [10].

Hughes has resolved equations governing two-dimensional flow of pedestrians derived for flows of both single and multiple pedestrian types. Similarly to some fluids flows, he has demonstrated the existence of two regimes of fluid, a high-density and a low-density regime [7].

Hughes has presented a good review about the crowd movement where the property of nonlinearity and time-dependence was investigated and the fact that crowd equations are conformably mappable. This property has made many interesting applications [3].

In reference [1], Xiaoping et al. have identified several methodological approaches for crowd evacuation. These approaches included models based on experiments with animals and used to propose several evacuation systems.

Al-Haboubi has proposed a new design to solve the safety problems about the congesting in Jamarat area. He has suggested to install uni-directional lanes and to restrict stoning to within a circular zone. An experiment was carried out to estimate the throwing time at a lane and used to estimate the waiting time in a simulation mode [11].

Fluid Models was proposed by Payne [12] and Whitham [13], where the basic hypothesis refers to the similarity between flow on roads and an incompressible fluid.

In a recent paper, Karamouzas et al. have presented a novel statistical-mechanical approach of crowd motion based on resemblance to interaction particle systems. Applied to a large collection of human motion data, this analysis reveals a simple power law interaction that is based not on the physical separation between pedestrians but on their projected time to a potential future collision, and is therefore fundamentally anticipatory in nature [14].

In this work, we propose a numerical model to study the flow of crowds at the Jamarat Bridge during the Hajj by assuming similarity between the crowd dynamics and a real incompressible fluid flow by means of computational fluid dynamics simulation ANSYS Fluent code. 
The objective of this study is to propose some modifications to the Jamarat Bridge to better manage the crowd movement and compare with the present Bridge.

Three modifications to the Jamarat Bridge domain are proposed. The velocity and the pressure for the flow are presented and compared to the present domain.

The paper is organized as follows: In Sections 2 and 3 the description and modelling of crowd dynamics problem is presented. Section 4 is devoted to present the details of the numerical resolution. The numerical parameters and algorithms are also presented in this section. In Section 5, discussions and comparisons about the proposed procedures are given.

\section{Description and Modelling of the Problem}

\subsection{Governing Equations}

In this work, we suppose that there is a similarity between crowd flow and the dynamics of an incompressible and laminar fluid. We investigate to study the crowd behavior in the Jamarat Bridge in the Hajj event where the people number is huge and the similarity is realistic. The governing equations of such a fluid are the classic Navier-Stokes equations. In the Cartesian two dimensional plane, the mass and momentum conservation equations are written as:

$$
\begin{gathered}
\frac{\partial u}{\partial x}+\frac{\partial v}{\partial x}=0 \\
\frac{\partial u}{\partial t}+u \frac{\partial u}{\partial x}+v \frac{\partial u}{\partial y}=-\frac{1}{\rho} \frac{\partial P}{\partial x}+v\left(\frac{\partial u^{2}}{\partial x^{2}}+\frac{\partial u^{2}}{\partial x^{2}}\right) \\
\frac{\partial u}{\partial t}+u \frac{\partial v}{\partial x}+v \frac{\partial v}{\partial y}=-\frac{1}{\rho} \frac{\partial P}{\partial y}+v\left(\frac{\partial v^{2}}{\partial x^{2}}+\frac{\partial v^{2}}{\partial x^{2}}\right)
\end{gathered}
$$

where $(u, v)$ is the velocity vector and $P$ is the pressure. The gravity effects are neglected here. $\rho$ and $v$ are density and kinematic viscosity of the fluid. The fluid used is the water, the density is taken $\rho=998 \mathrm{~kg} \cdot \mathrm{m}^{-3}$ and the kinematic viscosity is $v=1.0 \times 10^{-6} \mathrm{~m}^{2} \cdot \mathrm{s}^{-1}$.

\subsection{Description and Modelling of the Problem}

The partial differential equations governing the flow are discretized and resolved with the finite volume method. The computational domain is taken to be a part of the Jamarat Bridge, between the entry side of the Bridge to the part behind the trailing edge of the middle Jamarah.

In this work the third Jamarah is not included and the whole bridge is truncated to minimize the calculation cost. Figure 1 shows the sketch of the computational domain which is a $2 \mathrm{~d}$-representation of a part of the Jamarat Bridge.

To perform the equations resolution, the domain is meshed using a mesh generator software (GAMBIT package). The whole computational domain is divided into 4 blocks (Figure 1): Block1 1 (1-2-3-10-1), block 2 (10-3-4-9-10), block 3 (9-4-12-11-9) and block 4 (11-12-5-6-7-8-11). Block 1 is discretized with rectangular structured elements 


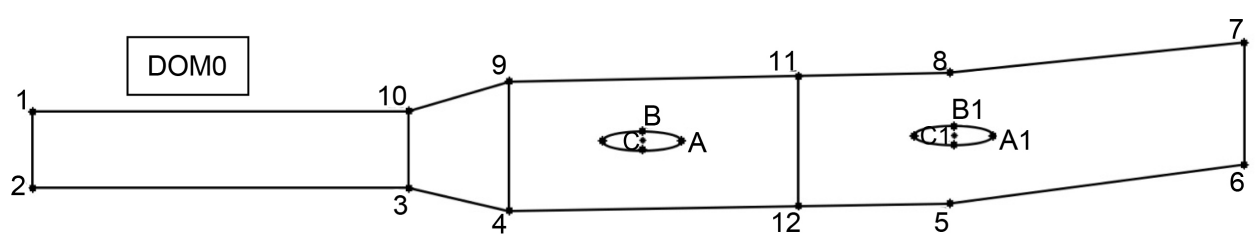

Figure 1. 2d-representation of the computational domain (DOM0) (a part of the Jamarat Bridge).

and all other blocks where the two pillars (Jamarat) are located, are discretized with triangular cells. The whole domain is divided on 365,000 cells. Figure 2 shows the grid near the two elements.

\section{Numerical Methodology}

The Ansys Fluent CFD code is used to resolve the Equations (1)-(3) governing the steady flow. In all investigated cases the inlet velocity is imposed initially to be horizontal and of value equal to $1.0 \mathrm{~m} / \mathrm{s}$. Second order central upwind discretization is used for the momentum equation. The time integration is done with an implicit method. The calculations are leaved until the residuals curves decrease down three decades and the pressure and velocity profiles don't vary.

The velocity is taken initially zero in all the boundary sides of the domain except the inlet and the outlet sides. At the inlet of the bridge, the velocity is constant and the outlet is set up as a pressure outlet. Boundary layers effect is not considered here.

The boundary conditions used in the simulation are recapitalized in Table 1.

\section{Results}

Three practical propositions are performed. The initial domain DOM0 (Figure 1) is modified to improve the velocity and pressure distributions. In the first modified domain DOM1 (Figure 3) points 10 and 8 and the points 3 and 5 are joined to suppress the region near sharp points 9 and 4 where fluid pressure can increase considerably.

The second proposed modification to the initial domain is to divide the whole domain into two subdomains DOM2 (Figure 3) with a longitudinal line. The objective is to better manage the dynamics of the flow behind the first Jamarah after stoning ritual.

In the third domain DOM3 proposed (Figure 3), the middle region between the two Jamarat where strong gradients and flow instability can occur, is reconstructed (Figure 3). Regular contours are designed using defined function of the form

$$
F(x)=A * \exp \left(-B * x^{2}\right)
$$

which provides a rather smooth curve. The leading edge and the trailing edge of shapes surrounding the first and second Jamarah are also modified to be more stretched. The major axis of the ellipse is taken greater. Figure 3 (DOM3) shows the size of the new shape versus the initial shape. 


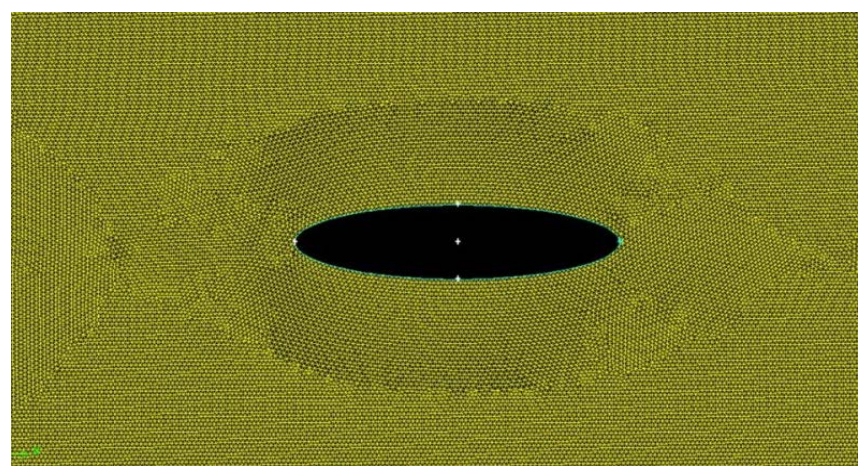

(a)

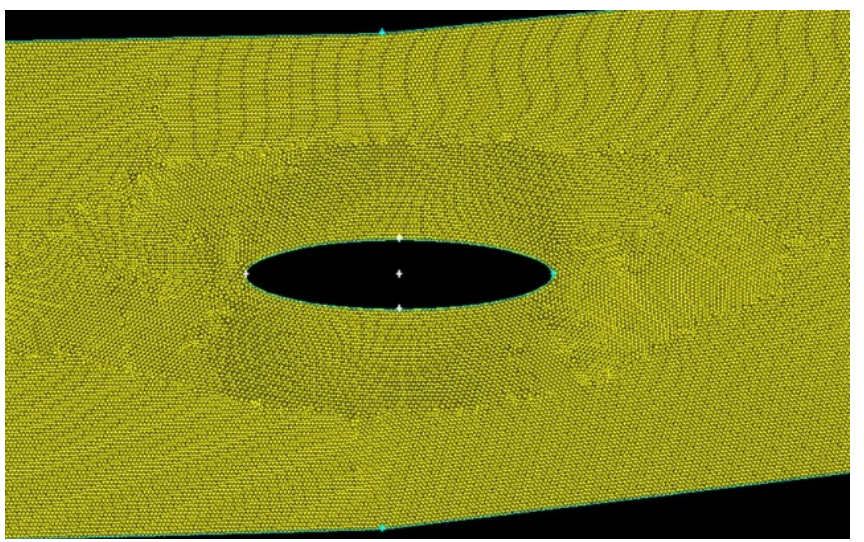

(b)

Figure 2. Grid distribution around the pillars (Jamarat): a. Little Jamarah, b. Middle Jamarah.
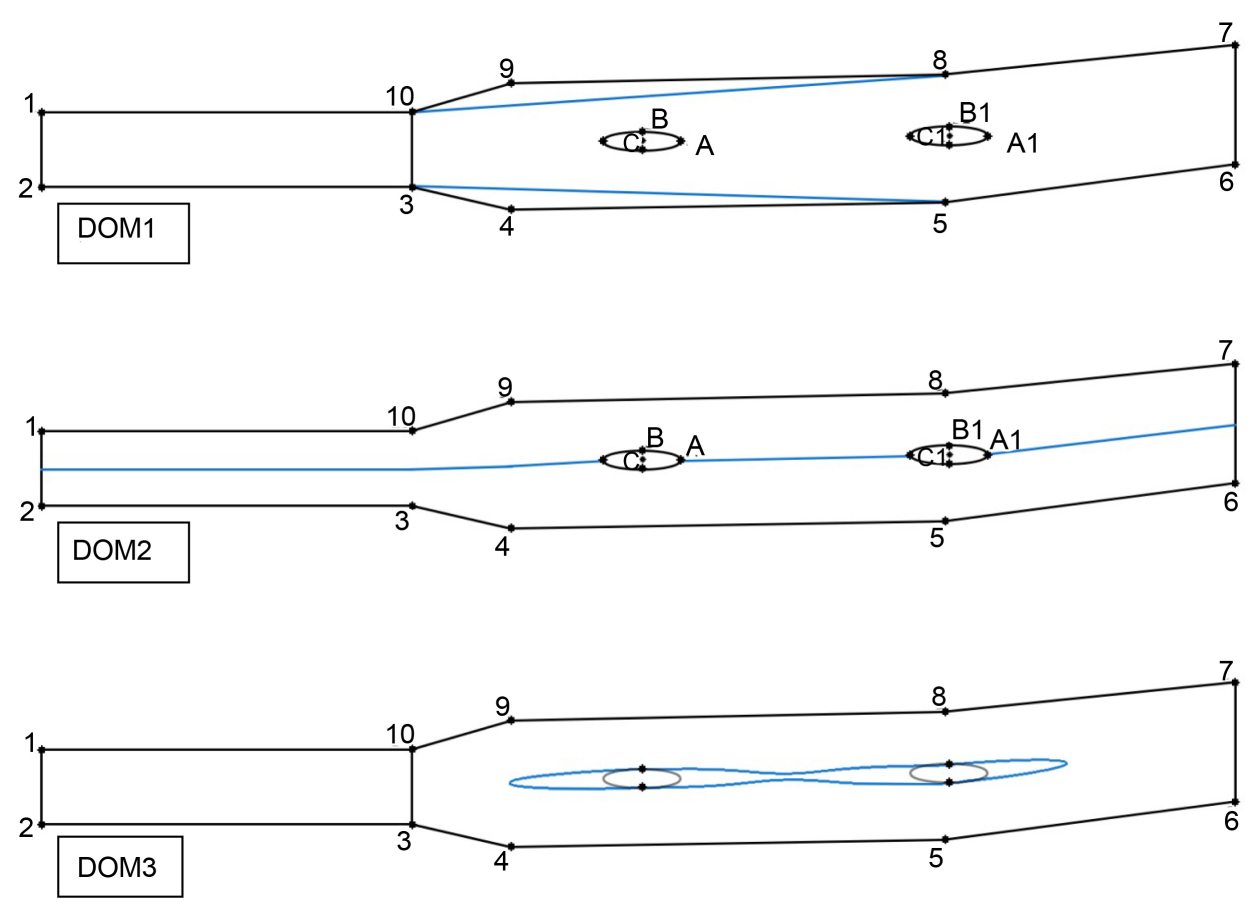

Figure 3. The proposed modified domains DOM1, DOM2, DOM3 (added obstacles colored). 
Table 1. Boundary conditions for the flow.

\begin{tabular}{ccccc}
\hline Inlet & Wall Boundary & Outlet & Boundary layers & Flow \\
\hline $\begin{array}{c}\text { Velocity inlet } \\
\text { Horizontal, } \mathrm{V}=1.0 \mathrm{~m} / \mathrm{s}\end{array}$ & No-slip & Pressure outlet & Not considered here & steady \\
\hline
\end{tabular}

In the three new domains proposed, Matlab and Photoshop software are used to design the shapes and exported in the mesh generator.

\section{Discussions}

The crowd flow can be fatal in sharp points especially when irregular and sudden rush occur. The leading edges of the shapes surrounding the Jamarat are high pressure side in the flow dynamics. The trailing edges of the Jamarat are sides where chaotic and turbulent flows can occur. The Three domains proposed in this work try to reduce the flow pressure, in particular in the critical points. Figure 4 shows the pressure contours in the (DOM1) (bottom) versus pressure initial contours distribution (top). Remarkable change in pressure behavior is observed which could be of great importance in the crowd movement.

In Figure 5 velocity contours in (DOM1) are represented. The flow in stopping points in the leading edge of the first Jamarah has good behavior compared to (DOM0). With the (DOM1) geometry, we can remark that only the flow dynamics are modified around the first Jamarat. To try to improve the behavior of the flow around the two Jamarat shapes, we have proposed to divide the whole domain as described above for the (DOM2). In Figure 6 and Figure 7 we present the pressure and velocity contours for the solution in the (DOM2, bottom figure) versus that of the (DOM0, top figure). As observed, a little improvement in the flow structure, nevertheless, this geometry can be beneficial to reduce the instability that can occur in the middle region from the trailing edge of the first Jamarah and the leading edge of the second because of reduction of the pedestrian number.

Figure 8 and Figure 9 show the flow behavior in (DOM3) versus the initial domain. The geometry of shapes are completely modified in order to separate between the pedestrian coming from the region around the first Jamarah after stoning the pillar and moving towards the second Jamarah. The pressure and velocity distributions are more regular. The fluid structure is more regular, in particular near the stopping points in the leading edges. The flow instabilities are less than the initial domain. The mathematical function used to design the shapes surrounding the two pillars (Jamarat) is promising and future investigation can better optimize this novel idea.

\section{Conclusion}

The problem of crowd dynamics in the Jamarat Bridge in Mina/Makkah was investigated in this paper with a model based on the likeness between collective crowd and the flow of a fluid. A CFD analysis of an incompressible laminar fluid flowing in 


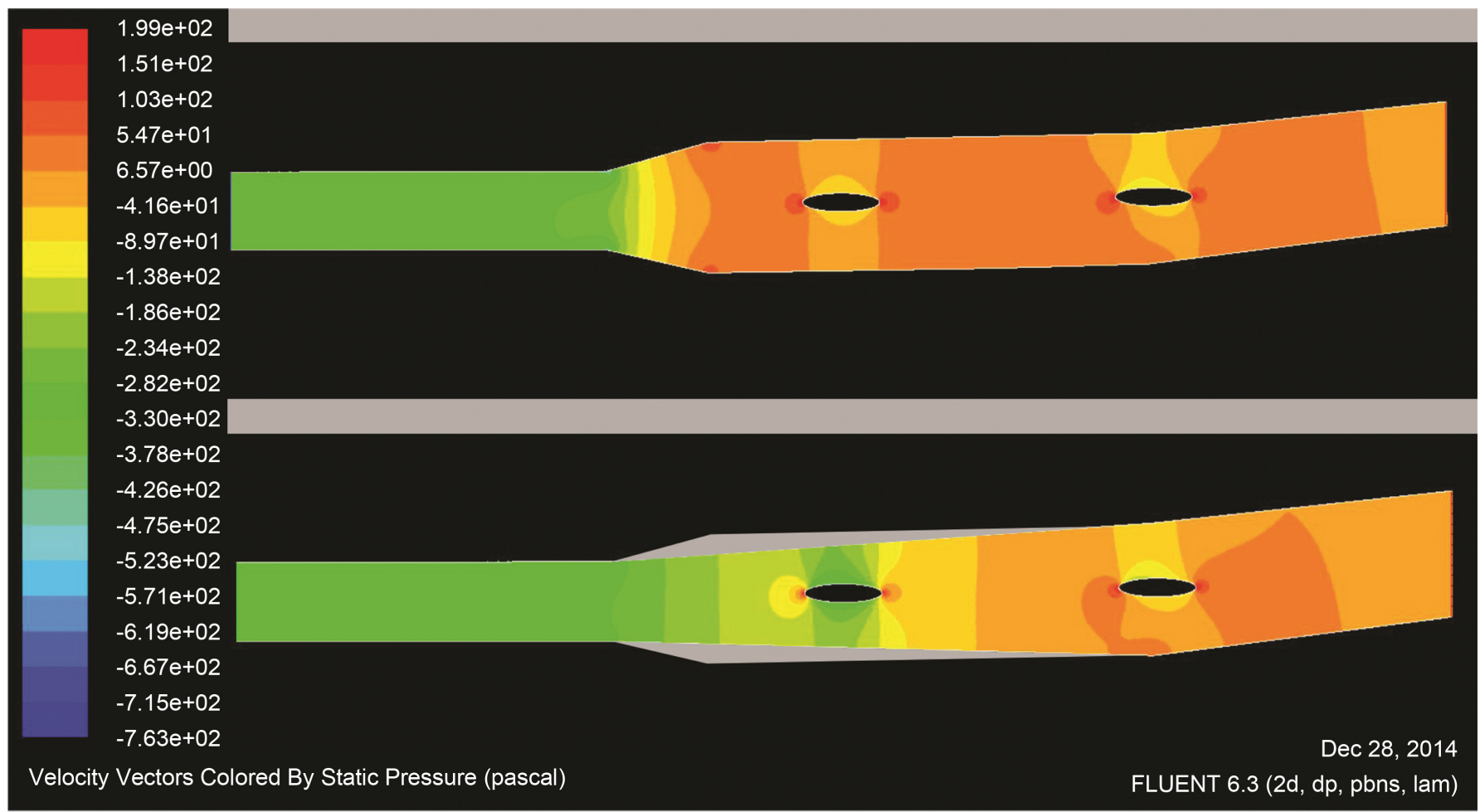

Figure 4. Pressure, top: initial domain DOM0, bottom: modified domain DOM1. (lower pressure around first Jamara, sharp points 10 and 3 show low pressure).

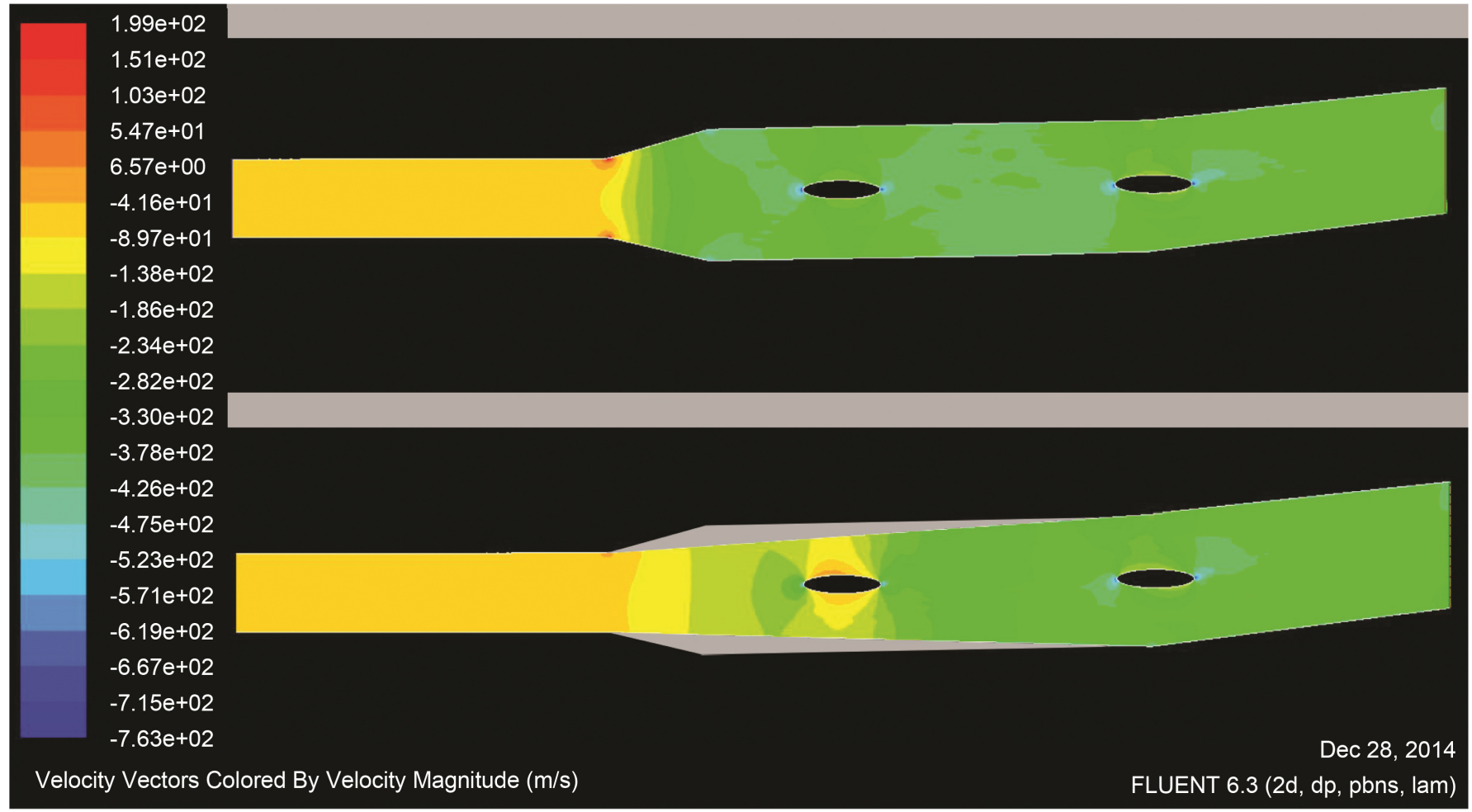

Figure 5. Velocity, top: initial domain DOM0, bottom: modified domain DOM1. (The flow structure around the second Jamarat is not modified). 


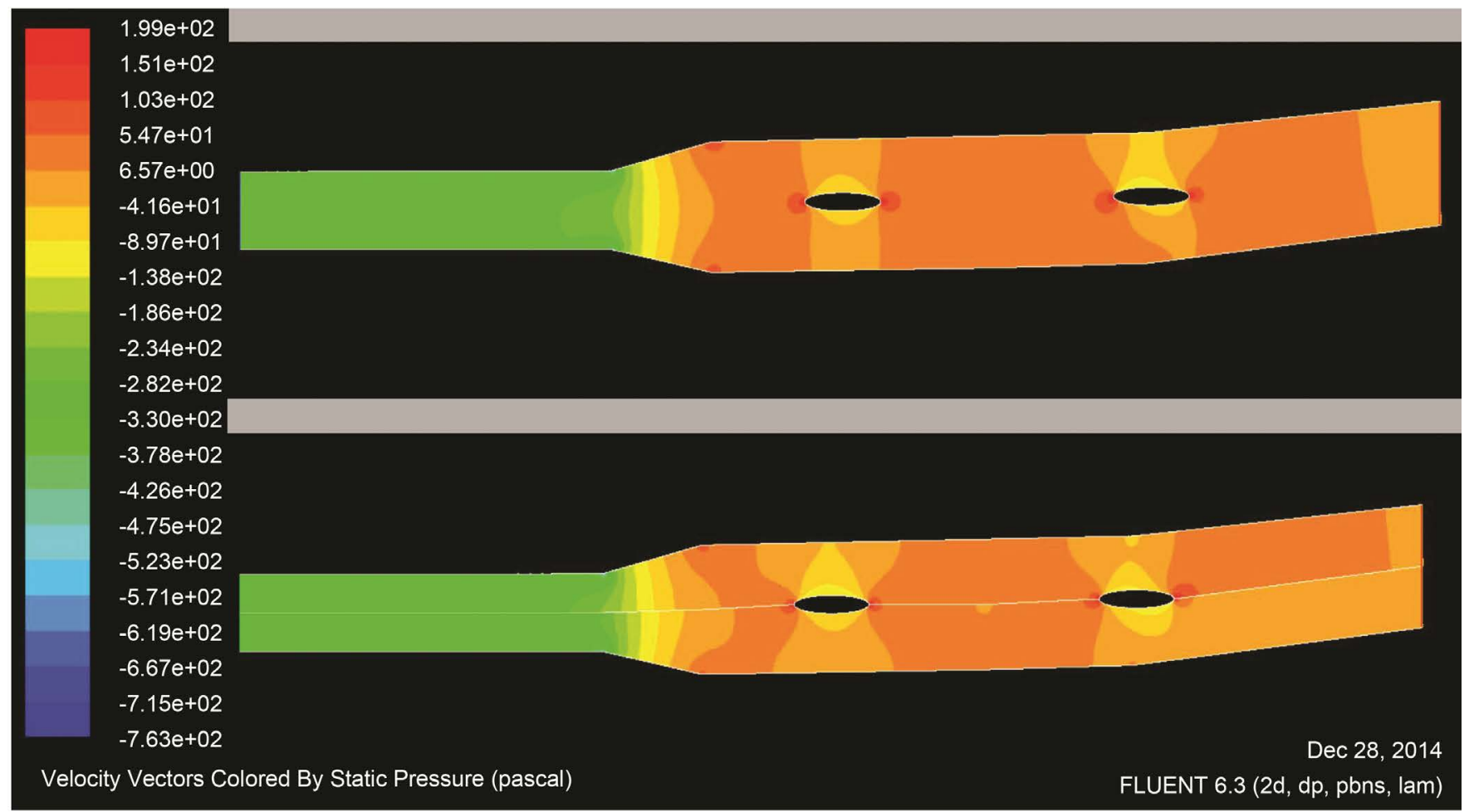

Figure 6. Pressure, top: initial domain DOM0, bottom: modified domain DOM2. (The geometry of DOM2 can be beneficial to reduce the instability in the middle region).

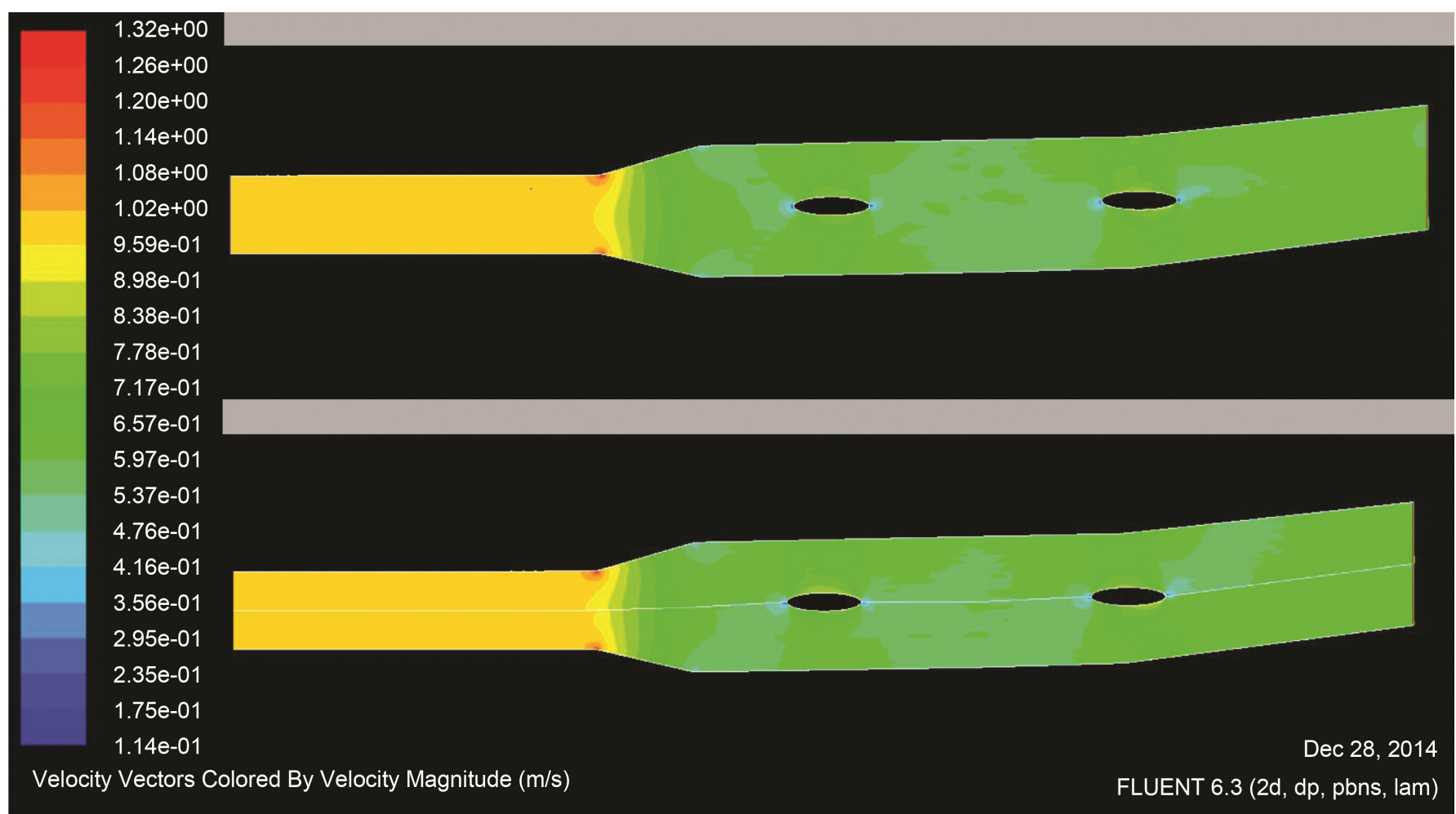

Figure 7. Velocity, top: initial domain DOM0, bottom: modified domain DOM2. (No significance change in the flow velocity in sharp or leading edge points). 


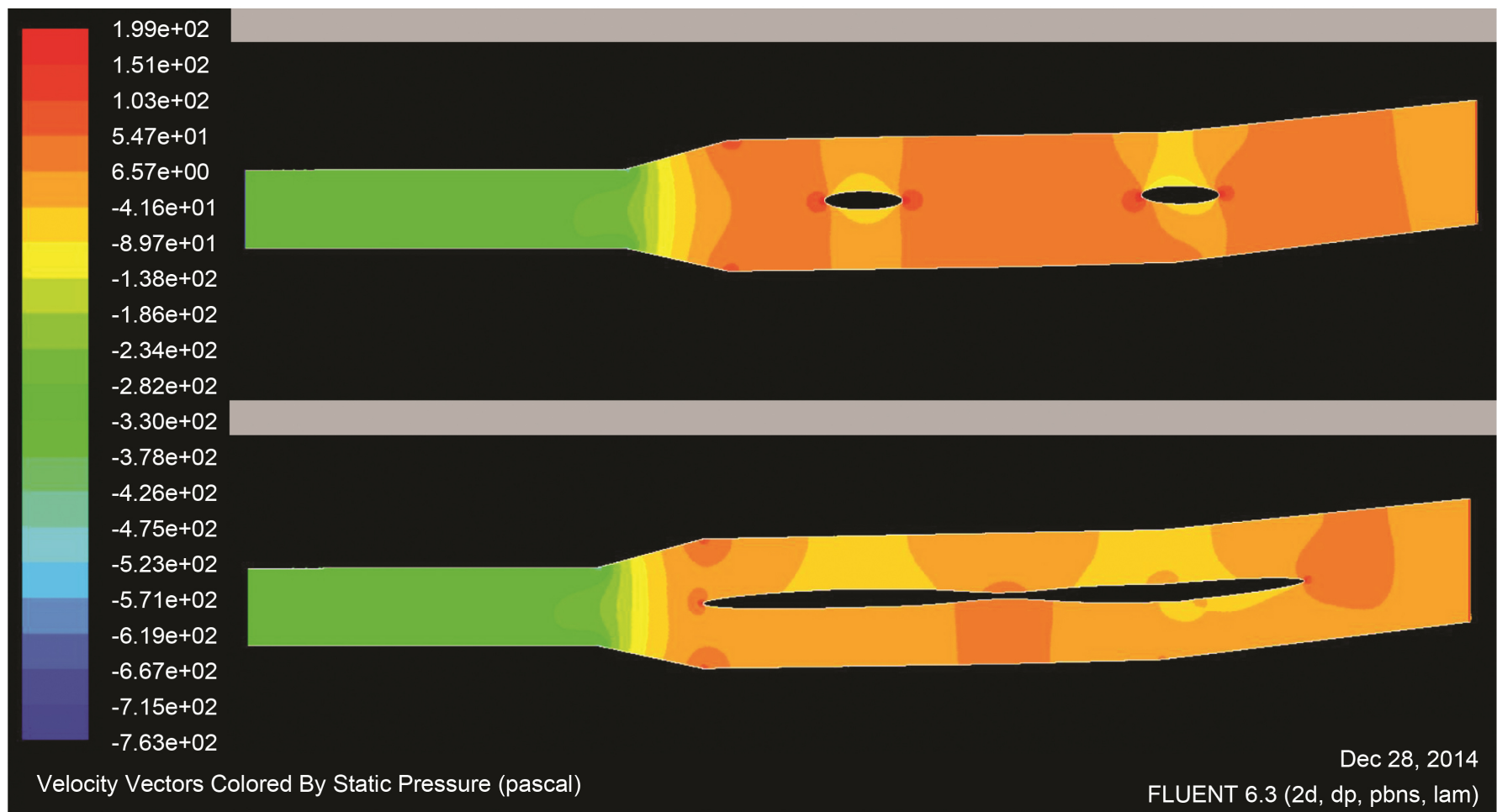

Figure 8. Pressure, top: initial domain DOM0, bottom: modified domain DOM3. (Separation between the pedestrian coming from the region around the first Jamarah and moving towards the second Jamarah, the fluid structure is more regular, Pressure is lower near the stopping points in the leading edges).

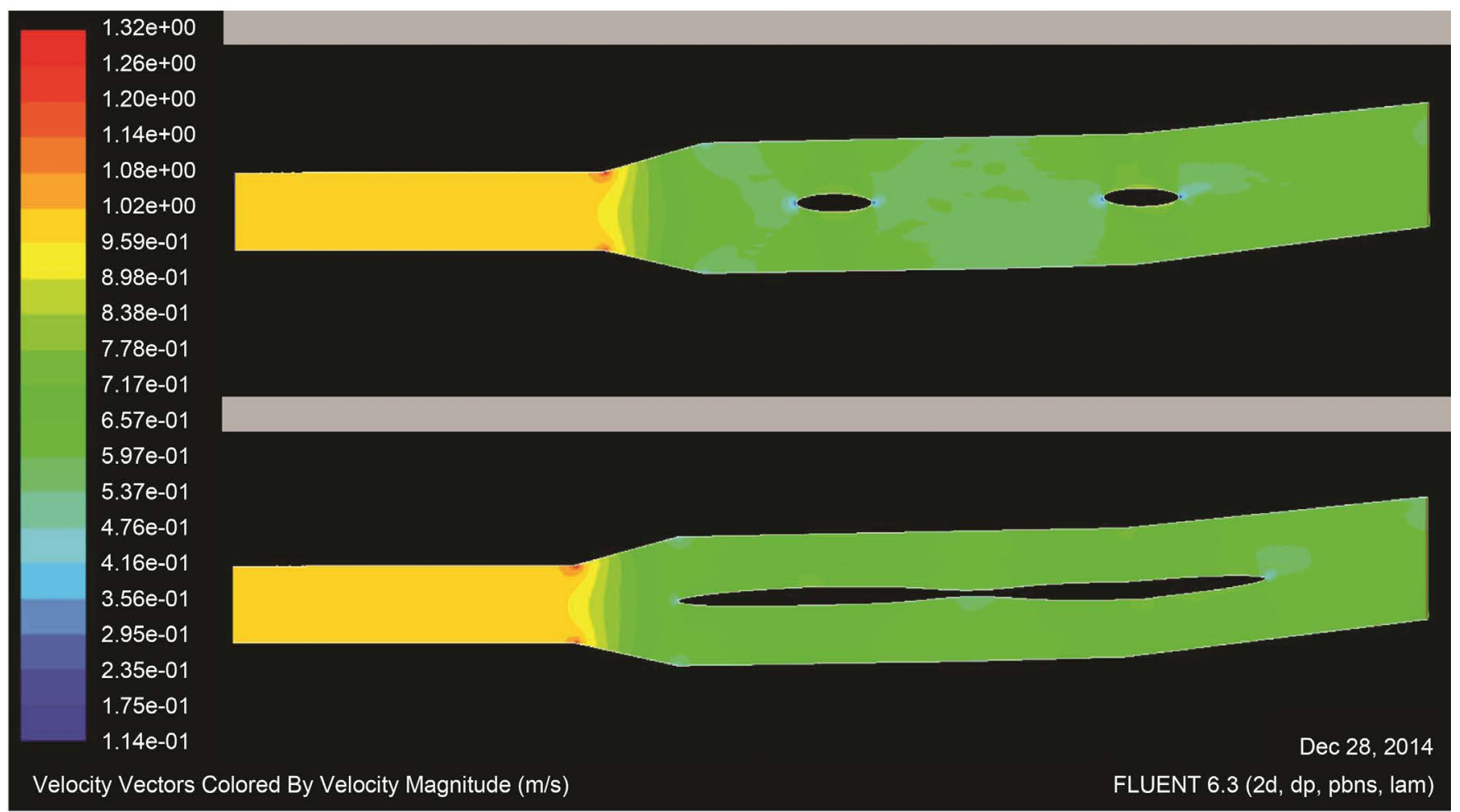

Figure 9. Velocity, top: initial domain DOM0, bottom: modified domain DOM3. (Less flow instabilities in the middle region). 
a 2D domain similar to the Jamarat Bridge is performed. Three modifications of the Bridge domain have been proposed and compared to the present domain. Comparisons of velocity and pressure behavior have been showed. The first proposition was to add obstacles to eliminate sharp points in the bridge border before reaching the first Jamarah.

The second proposal is to divide the bridge into two sub-domains with a longitudinal barrier throughout the whole domain. The third idea is to modify the form of the Jamarat contours in aim to eliminate the space between them by a fairly regular line and reduce flow instabilities. The barriers that can be added to the bridge may be permanent or temporary.

With the fluid simulations on three new domains, the three solutions showed good behavior for the velocity and pressure functions. The stopping points and strong gradients of the crowd movements can be avoided and improved.

\section{Acknowledgements}

Authors would like to thank the deanship of scientific research at King Faisal University (KFU), Alhasa, Saudi Arabia for supporting this research (Grant number 165040).

\section{References}

[1] Zheng, X.P., Zhong, T.K. and Liu, M.T. (2009) Modeling Crowd Evacuation of a Building Based on Seven Methodological Approaches. Building and Environment, 44, 437-445. https://doi.org/10.1016/j.buildenv.2008.04.002

[2] Bellomo, N. and Dogbe, C. (2011) On The Modeling of Traffic and Crowds: A Survey of Models, Speculations, and Perspectives. SIAM Review, 53, 409-463. https://doi.org/10.1137/090746677

[3] Hughes, R.L. (2003) The Flow of Human Crowds. Annual Review of Fluid Mechanics, 35, 169-182. https://doi.org/10.1146/annurev.fluid.35.101101.161136

[4] Cusack, M.A. (2002) Modelling Aggregate-Level Entities as a Continuum. Mathematical and Computer Modelling of Dynamical Systems, 8, 33-48.

https://doi.org/10.1076/mcmd.8.1.33.8339

[5] Helbing, D.A. (1992) Fluid Dynamic Model for the Movement of Pedestrians. Complex Systems, 6, 391-415. http://arxiv.org/abs/cond-mat/9805213v1

[6] Hughes, R.L. (2000) The Flow Of Large Crowds Of Pedestrians. Mathematics and Computers in Simulation, 53, 367-370. https://doi.org/10.1016/S0378-4754(00)00228-7

[7] Hughes, R.L. (2002) A Continuum Theory for the Flow of Pedestrians. Transportation Research Part B, 36, 507-535. https://doi.org/10.1016/S0191-2615(01)00015-7

[8] Smith, R.A. (1995) Density, Velocity and Flow Relationships for Closely Packed Crowds. Safety Science, 18, 321-327. https://doi.org/10.1016/0925-7535(94)00051-4

[9] Henderson, L.F. (1974) On the Fluid Mechanics of Human Crowd Motion. Transportation Research, 8, 509-515. https://doi.org/10.1016/0041-1647(74)90027-6

[10] Helbing D., Johansson A. and Al-Abideen H.Z. (2007) The Dynamics of Crowd Disasters: An Empirical Study. Physical Review E, 75, 046109. https://doi.org/10.1103/physreve.75.046109 
[11] Al-Haboubi, M.H. (2003) A New Layout Design for the Jamarat Area (Stoning the Devil), Arabian Journal for Science and Engineering, 28, 131-142.

[12] Payne, H.J. (1971) Models of Freeway Traffic and Control. In: Bekey, G.A., Ed., Mathematical Models of Public Systems, Simulation Council, La Jolla, Vol. 1, 51-61.

[13] Whitham, G.B. (1999) Linear and Nonlinear Waves. Wiley, New York. (reprint of the 1974 original, a Wiley-Interscience publication)

[14] Karamouzas, L., Skinner, B. and Guy, S.J. (2014) A Universal Power Law Governing Pedestrian Interactions. Physical Review Letters, 113, Article ID: 238701.

https://doi.org/10.1103/PhysRevLett.113.238701

Submit or recommend next manuscript to SCIRP and we will provide best service for you:

Accepting pre-submission inquiries through Email, Facebook, LinkedIn, Twitter, etc.

A wide selection of journals (inclusive of 9 subjects, more than 200 journals)

Providing 24-hour high-quality service

User-friendly online submission system

Fair and swift peer-review system

Efficient typesetting and proofreading procedure

Display of the result of downloads and visits, as well as the number of cited articles

Maximum dissemination of your research work

Submit your manuscript at: http://papersubmission.scirp.org/

Or contactojfd@scirp.org 